\title{
Local cosmic string and C-field
}

\section{F.Rahaman*,R.Mondal* and M.Kalam**}

\begin{abstract}
We investigate a local cosmic string with a phenomenological energy momentum tensor as prescribed by Vilenkin, in presence of C-field . The solutions of full nonlinear Einstein's equations for exterior and interior regions of such a string are presented.
\end{abstract}

\section{INTRODUCTION}

An extremely interesting and attractive feature of gauge theories is the topological defects associated with spontaneous symmetry breaking[1]. A large amount of the structure of the known Universe may have resulted from the formation of a type of topological defects known as a cosmic string [2]. It is assumed that at very early stages of its evolution, the Universe has gone through a number of phase transitions and each of which could have resulted in the formation of topological defects, which may be all quite different in character. These include point like defects known as monopoles, string like defects ( cosmic strings) and domain walls which are sheet like defects[3]. Among these defects cosmic strings are particularly interesting since they may have played relevant cosmological roles, such as, for examples, large scale structures or galaxy formation and its detection can be an observational confirmation of the standard theory [4]. Strings are said to be local or global depending on their origin from the breakdown of local or global $\mathrm{U}(1)$ symmetry. The local strings are well behaved, having an exterior representing a flat Minkowskian space-time with a conical defect. In recent past, Sen et al [5] and Arazi et al [6] have shown that a local string with a phenomenological energy momentum tensor, as prescribed by Vilenkin [7] was inconsistent in Brans-Dicke theory. In last few decades there has been considerably interest in alternative theories of gravity. One of the important alternative theory is C-field theory introduced by Hoyle and Narlikar [HN] [8]. $\mathrm{HN}$ adopted a field theoretic approach introducing a mass-less and charge-less scalar field

\footnotetext{
${ }^{0}$ Pacs Nos : 04.20.Gz;04.50.+h

Key words and phrases : Local cosmic string, C-field $*$ Dept.of Mathematics,Jadavpur University,Kolkata-700 032,India ** Dept. of Phys. , Netaji Nagar College for Women, Regent Estate, Kolkata-700092,India E-Mail:farook_rahaman@yahoo.com
} 
$\mathrm{C}$ in the Einstein - Hilbert action to account for the matter creation. A C - field generated by a certain source equation, leads to interesting changes in the cosmological solution of Einstein field equations. As far as our knowledge there has not been any work in literature where C-field is introduced to study local string. In this paper, we would like to study exterior and interior solutions of the local string in presence of C-field and look forward how the local string solutions are effected by $\mathrm{C}$ - field.

\section{BASIC EQUATIONS:}

The modified Einstein equation due to HN through the introduction of an external C field are[8]

$$
R^{a b}-\frac{1}{2} g^{a b} R=-8 \pi G\left[T^{a b}-f C^{a} C^{b}+\frac{1}{2} f g^{a b} C^{i} C_{i}\right]
$$

where $\mathrm{C}$, a scalar field representing creation of matter, $x_{i}, \mathrm{i}=0,1,2,3$ stand for the space-

time coordinates with $C_{i}=\frac{\partial C}{\partial x_{i}}$ and $T_{a b}$ is the matter tensor and 'f' is a positive coupling constant. The general static cylindrically symmetric metric

$$
d s^{2}=e^{2(K-U)}\left(-d t^{2}+d r^{2}\right)+e^{2 U} d z^{2}+W^{2} e^{-2 U} d \theta^{2}
$$

is taken to describe the space-time given by an infinitely long static local string with the axis of symmetry being $\mathrm{z}$ axis . $\mathrm{K}, \mathrm{U}$ and $\mathrm{W}$ are functions of the radial coordinate ' $r$ ' alone. The local string is characterized by an energy density and a stress along the symmetry axis given by

$$
T_{t}^{t}=T_{z}^{z}=-\sigma
$$

and all other components are zero[7].

The field equations can be written as

$$
\begin{gathered}
-\frac{W^{\prime \prime}}{W}+\frac{K^{\prime} W^{\prime}}{W}-U^{\prime 2}=-8 \pi G \sigma e^{2(K-U)}+8 \pi G f C^{\prime 2} \\
-\frac{K^{\prime} W^{\prime}}{W}+U^{\prime 2}=-8 \pi G f C^{\prime 2} \\
-K^{\prime \prime}-U^{\prime 2}=8 \pi G f C^{\prime 2} \\
-\frac{W^{\prime \prime}}{W}+2 \frac{U^{\prime} W^{\prime}}{W}-U^{\prime 2}+2 U^{\prime \prime}-K^{\prime \prime}=-8 \pi G \sigma e^{2(K-U)}+8 \pi G f C^{\prime 2}
\end{gathered}
$$


where a prime represents differentiation w.r.t. 'r'. Here we assume that creation field C depends on radial coordinate 'r' only.

\section{EXTERIOR SOLUTIONS:}

Now we shall find the metric in the exterior of the source by solving the above equations for vacuum i.e. with $\sigma=0$. From equations (4) and (7), we get

$$
K^{\prime}-2 U^{\prime}=\frac{b}{W}
$$

Also from equations (5) and (6), we get

$$
K^{\prime}=\frac{a}{W}
$$

[ a,b are integration constants]

Equations (8) and (9) now combine to yield the equation

$$
U^{\prime}=\frac{a-b}{2 W}
$$

From equations (4) and (5), one gets

$-\frac{W^{\prime \prime}}{W}=0$

This implies

$$
W=c r+d
$$

[ c,d are integration constants]

Now using (11), one can integrate equations (9) and (10) to yield

$$
\begin{gathered}
K=\frac{a}{c} \log \left[K_{0}\left(r+\frac{d}{c}\right)\right] \\
U=\frac{(a-b)}{2 c} \log \left[U_{0}\left(r+\frac{d}{c}\right)\right]
\end{gathered}
$$


[ $U_{0}$ and $K_{0}$ are integration constants and can be chosen as unity without any loss of generality ]

Also we get an expression for $\mathrm{C}$ from the field equations as

$$
C=\sqrt{\left[\frac{a c-\left(\frac{a-b}{2}\right)^{2}}{8 \pi G f c^{2}}\right]} \log \left[C_{0}\left(r+\frac{d}{c}\right)\right]
$$

[ $C_{0}$ is an integration constant]

\section{INTERIOR SOLUTIONS:}

We shall now focus on interior solutions. The field equations (4) - (7) are a system of four equations with five unknown parameters $\mathrm{U}, \mathrm{W}, \mathrm{K}, \sigma$ and $\mathrm{C}$. One additional constraint relating these parameters is required to obtain explicit solutions of the system. Following Rahaman et al [9], we assume $C^{\prime}=$ constant $=Q($ say $)$, for simplicity, to obtain unique solution of the field equations. From field equations (5) and (6), we get

$$
K^{\prime}=\frac{A}{W}
$$

Also from equations (4) and (7), one gets

$$
K^{\prime}-2 U^{\prime}=\frac{B}{W}
$$

[ A,B are integration constants]

From equation (5) by using (15) and (16), we get

$$
W^{\prime}=E+D W^{2}
$$

where

$$
E=\frac{(A-B)^{2}}{4 A}, D=\frac{8 \pi G f Q^{2}}{A}
$$


Solving (17), one gets

$$
W=\sqrt{\left(\frac{E}{D}\right)} \tan \sqrt{E D}\left(r+r_{0}\right)
$$

[ $r_{0}$ is an integration constant]

Also from equations (15) and (16), one finds the solutions of $\mathrm{U}$ and $\mathrm{K}$ as

$$
\begin{gathered}
K=\left(\frac{A}{E}\right) \log \left[K_{0} \sin \sqrt{E D}\left(r+r_{0}\right)\right] \\
U=\frac{(A-B)}{2 E} \log \left[U_{0} \sin \sqrt{E D}\left(r+r_{0}\right)\right]
\end{gathered}
$$

$\left[U_{0}\right.$ and $K_{0}$ are integration constants and one could take these constants to be unity , without any loss of generality ]

The string energy density is given by

$$
\sigma=\frac{E D}{4 \pi G}\left[\sec \sqrt{E D}\left(r+r_{0}\right)\right]^{2}\left[\csc \sqrt{E D}\left(r+r_{0}\right)\right]^{\frac{A+B}{E}}
$$

\section{DISCUSSIONS:}

In this work, we have shown that local cosmic string is consistent in C-field theory. We have found the exterior and interior solutions for the metric of static local cosmic string. The interior solutions obtained in the present work are no means the general ones, but it explicitly exhibits a consistent set of interior solutions of the non linear field equations for a local cosmic string in C-field theory. The complete solution for the metric for the interior of the string is

$d s^{2}=[\sin \sqrt{E D}(r)]^{\frac{(A+B)}{E}}\left(-d t^{2}+d r^{2}\right)+[\sin \sqrt{E D}(r)]^{\frac{(A-B)}{E}} d z^{2}+\frac{E}{D} \tan ^{2} \sqrt{E D}(r)[\sin \sqrt{E D}(r)]^{\frac{(B-A)}{E}} d \theta^{2}$

One should note that for $r \rightarrow 0$ i.e. near the axis of the string, the line element becomes

$$
d s^{2}=A_{0} r^{\frac{A+B}{E}}\left(-d t^{2}+d r^{2}\right)+B_{0} r^{\frac{A-B}{E}} d z^{2}+D_{0} r^{2+\frac{B-A}{E}} d \theta^{2}
$$

[ Taking $r_{0}$ equal to zero and $A_{0}, B_{0}$ and $D_{0}$ are constants ] 
Now if we put $\frac{A}{E}=k^{2}$ and $\frac{B}{E}=k(k-2)$, then the metric $(24)$ can be written as

$$
d s^{2}=r^{2\left(k^{2}-k\right)}\left(-d t^{2}+d r^{2}\right)+r^{2 k} d z^{2}+D_{0} r^{2(1-k)} d \theta^{2}
$$

Thus one can get, one parameter family of solution. To understand the meaning and behavior of the metric (23), one needs to match it with exterior solutions (11) - (13). For the general metric (23), the curvature scalar is given by

$$
R=[\csc \sqrt{E D}(r)]^{\frac{(A+B)}{E}} \sqrt{\left(\frac{D}{E}\right)} \cot \sqrt{E D}(r)\left[4 E D-2(A D) \sec ^{2} \sqrt{E D}(r)\right]
$$

Thus, apparently, it seems from (26) that, space-time becomes singular periodically at finite distances from the axis of the string. But, one can not take large ' $r$ ' so that other singularity will develop except for $r=0$. From metric (23), it is evident that apart from $r=0$, there are other coordinates singularities will arise at $r=\frac{n \pi}{\sqrt{E D}}(>>$ so called $\epsilon)$

[ 'n' is an integer ]

If one assumes the thickness of the string is $<\frac{\pi}{\sqrt{E D}}$ and as the metric (23) represents the interior spacetime of the cosmic string, so one can not take the value of $r \geq \frac{\pi}{\sqrt{E D}}$. So further singularity could not arise.

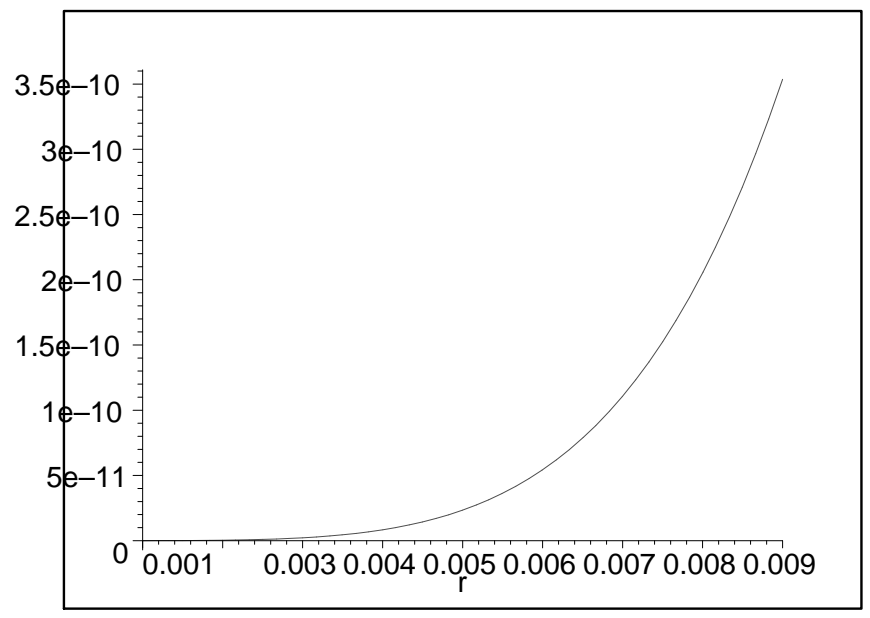

Figure 1: Diagram of the metric coefficient $g_{t t}$ for $A=21, B=1, D=\frac{21}{100}, E=\frac{100}{21}$

To have an idea of the motion of test particles, one can calculate the radial acceleration vector $A^{r}$ of a particle that remains stationary ( i.e $V^{1}=V^{2}=V^{3}=0$ ) in the field of the string [5]. Let us consider an observer with four velocity $V_{i}=\sqrt{\left(g_{00}\right)} \delta_{i}^{t}$. Now $A^{r}=V_{; 0}^{1}=\Gamma_{00}^{1} V^{0} V^{0}$. 
Hence the line element (23), we have

$$
A^{r}=\sqrt{\frac{D(A+B)^{2}}{4 E}}[\cot \sqrt{E D}(r)][\csc \sqrt{E D}(r)]^{\frac{(A+B)}{E}}
$$

Hence one can see that the gravitational force varies with the radial distance and also $A^{r}>0$. So the particle has to accelerate away from the string, which implies that gravitational field due to string is attractive[5]. The solutions obtained here are important as they are perhaps the first analytical solutions for a local string in presence of C-field. A detail analysis by taking $C^{\prime} \neq 1$ will certainly give more insight of various aspects of local string solution in presence of C-field. Work in this direction is in progress and could be noted else where.

\section{Acknowledgements}

F.R is thankful to Jadavpur University and DST, Government of India for providing financial support under Potential Excellence and Young Scientist scheme. We are grateful to anonymous referee for his several critical remarks and constructive suggestions, which has led to a stronger result than the one in the original version.

\section{References}

[1] Sakellariadou M arxiv: hep-ph/ 0212365

[2] Hindmarsh M D and Kibble T W B Rep. Prog. Phys. 58, 477 )(1995)

[3] A.Vilenkin and E.P.S. Shellard (1994), Cosmic String and other Topological Defects (Camb. Univ. Press) Cambridge.

[4] Shirasaki E Y et al arxiv: astro-ph/ 0305353

[5] Sen A A and Banerji N and Banerji A Phys.Rev.D(1997) 56,3706

[6] Arazi A and Simeone C (2000) Gen.Rel.Grav. 32, 2259

[7] Vilenkin A (1985) Phys. Rep. 121, 263

[8] Hoyle. F and Narlikar. J. V. Proc.Roy.Soc. A 290 (1966) 162 ; Narlikar. J. V. An introduction to Cosmology, (Camb.Univ.Press), Cambridge (2002)

[9] F Rahaman, B C Bhui and R Mukherji Chinese J. of Physics 43, 806 ( 2005 ) 